\title{
State Estimation Methods for Sound Environment System Based on a Fuzzy Probability Theory
}

\author{
Akira Ikuta, ${ }^{1}$ Hisako Masuike ${ }^{2}$ and Natsuko Hayakawa ${ }^{3}$ \\ ${ }^{1}$ Department of Management Information Systems, Prefectural University of \\ Hiroshima, 1-1-7l Ujina-Higashi, Minamiku, Hiroshima, 734-8558; ${ }^{2}$ System \\ Development Division, NTT DATA Chugoku Co., 11-20 Hijiyama, Minamiku, \\ Hiroshima, 732-0816; ${ }^{3} \mathrm{c} / \mathrm{o}$ Prof Akira Ikuta, Graduate Course of Human Life \\ and Environmental Science, Hiroshima Prefectural Women's University, \\ 1-1-71 Ujina-Higashi, Minamiku, Hiroshima, 734-8558; Japan.
}

\begin{abstract}
In the measurement and evaluation of actual random phenomena in sound environment systems, the observed data exhibit various probability distribution forms, and often contain fuzziness due to the existence of confidence limitation in measuring instruments, permissible error in experimental data, and the variety of human response to phenomena, etc. In this study, by introducing fuzzy probability theory, two types of state estimation methods based on the fuzzy observations are theoretically proposed from both the static and dynamic viewpoints. First, a static method for estimating the probability distribution of a signal in the psychological evaluation for sound environment is discussed theoretically by considering the human noise annoyance scores as observation data with fuzziness. Next, a dynamical estimation method for a signal in sound environment systems based on fuzzy observations is theoretically proposed under the actual situation of existence of an external noise. The validity and effectiveness of the proposed methods are experimentally confirmed by applying the theories to the actual fuzzy data observed in a sound environment
\end{abstract}

Reprint requests to: Professor Akira Ikuta, Department of Management Information Systems, Prefectural University of Hiroshima, 1-1-71 Ujina-Higashi, Minamiku, Hiroshima, 734-8558 Japan.ikuta@pu-hiroshima.ac.jp 


\section{KEYWORDS}

State estimation, fuzzy probability, sound environment, psychological evaluation, fuzzy observation, noise annoyance

\section{INTRODUCTION}

In the sound environment systems related to the mutual effects on physical phenomenon and human response, most of the actual observed data show a complex fluctuation pattern differing from a standard Gaussian distribution, and furthermore, these often contain the fuzziness due to the existence of external noise, permissible error in experimental data, and the variety of human response to phenomena. Therefore, the fluctuation waveform of the specific signal must be momentarily estimated as precisely as possible, based on the observed data with fuzziness, in order to evaluate the phenomena.

Many standard estimation methods proposed previously in the field of stochastic system theory have not considered positively the fuzziness in the observation data under the restriction of Gaussian type fluctuation (in most cases, zero mean), for the simplification of theory (Kalman, 1960; Kalman and Bucy, 1961; Kushner, 1967). Although several state estimation methods for a stochastic environment system with non-Gaussian fluctuations have previously been proposed (Ohta, et al. 1976; Ohta and Yamada, 1984), the fuzziness contained in the observed data has not been considered in these studies. Therefore, it is desirable to develop a method that is flexible and is applicable to ill-conditioned fuzzy observations.

In this paper, by using fuzzy probability (Zadeh, 1968; Tamaki et al., 1992), new estimation theories are proposed for a signal based on fuzzy observations with non-Gaussian properties, from static and dynamic viewpoints. A static method for the estimation of the probability density function of the signal is proposed first for the psychological evaluation in a sound environment. More specifically, by regarding the human noise annoyance scores as.observation data with fuzziness, an estimation method of the probability density function of the sound level with nonGaussian properties is proposed on the basis of the observations of noise annoyance scores. Next, a dynamical state estimation method, based on the observed data containing the fuzziness and the effects of an external noise with non-Gaussian type is proposed in a recursive form suitable for use with a digital computer. After paying 
attention to the power state variable for a specific signal in a sound environment, which exhibits complex probability distribution forms, by introducing a new type of membership function for the power state variable, a state estimation method is theoretically derived. A Gamma distribution is suitable to represent the power state variable, which fluctuates only within the positive region. The proposed digital filter positively utilizes the additive property of power state variables in the estimation algorithm. Finally, the proposed theories are applied to the actual estimation problems of the sound environment, and the usefulness is experimentally verified.

\section{STATIC STATE ESTIMATION METHOD FOR PSYCHOLOGICAL EVALUATION OF SOUND ENVIRONMENT}

To find the quantitative relationship between the human noise annoyance and the physical sound level for environmental noises is important from the viewpoint of noise assessment. Especially, in the evaluation for a regional sound environment, the investigation based on questionnaires to the regional inhabitants is often given when the experimental measurement at every point in the whole area of the region is difficult. Therefore, it is very important to derive the mutual relationship between noise annoyance and sound level from a statistical viewpoint connected closely with the investigation through questionnaires and noise evaluation quantities: $L_{e q}$ (averaged energy on decibel scale), $L_{\alpha}((100-\alpha)$ percentile level; a level that the cumulative distribudion of the sound level shows $1=\alpha / 100, \alpha=5,10,50,90,95$ ), and $L_{\max }$ (a maximum level of the fluctuating sound level within a time period)etc.

Consider a sound environment system with an arbitrary distribution of nonGaussian type. The probability density function of a specific signal $x$ in the sound level can be expressed in an expansion series form as (Ohta \& Nakagami, 1957; Ohta \& Koizumi, 1968):

$$
\begin{aligned}
& P(x)=\frac{1}{\sqrt{2 \pi \sigma_{x}^{2}}} e^{-\frac{\left(x-\mu_{x}\right)^{2}}{2 \sigma_{x}^{2}}}\left\{1+\sum_{n=1}^{\infty} A_{n} \frac{1}{\sqrt{n !}} H_{n}\left(\frac{x-\mu_{x}}{\sigma_{x}}\right)\right\}, \\
& \mu_{x}=<x>, \sigma_{x}^{2}=<\left(x-\mu_{x}\right)^{2}>, \\
& A_{n}=<\frac{1}{\sqrt{n !}} H_{n}\left(\frac{x-\mu_{x}}{\sigma_{x}}\right)>,(n \geq 3), A_{0}=1, A_{l}=A_{2}=0,
\end{aligned}
$$


The function $H_{n}(x)$ in Eq.(1) is Hermite polynomial of $n$-th order defined by (Cramer, 1951)

$$
H_{n}(x)=(-1)^{n} e^{x^{2} / 2} \frac{a^{-n}}{d x^{n}} e^{-x^{2} / 2}
$$

and satisfies the following orthonormal condition:

$$
\int_{-\infty} \frac{1}{\sqrt{2 \pi \sigma_{x}^{2}}} e^{-\frac{\left(x-\mu_{x}\right)^{2}}{2 \sigma_{x}^{2}}} \frac{1}{\sqrt{n !}} H_{n}\left(\frac{x-\mu_{x}}{\sigma_{x}}\right) \frac{1}{\sqrt{m !}} H_{m}\left(\frac{x-\mu_{x}}{\sigma_{x}}\right) d x=\delta_{n m},
$$

where $\delta_{n m}$ denotes Kronecker's delta. Furtermore, $<>$ denotes the averaging operation on the variables, and when the data $x_{1}, x_{2}, \ldots, x_{N}$ of a variable $x$ are obtained it is defined by

$$
<f(x)>=\frac{1}{N} \sum_{i=1}^{N} f\left(x_{i}\right),
$$

for arbitrary function $f(x)$. Two parameters $\mu_{-}$and $\sigma_{x}^{2}$ denote the mean and variance of $x$, and $A_{n}(n>3)$ are expansion coefficients reflecting the higher order statistics.

Let the psychological evaluation value by use of the human judgment method (Namba et al., 1978) based on 7 scores (1. very calm, 2. calm, 3. mostly calm, 4. little calm, 5 . noisy, 6. fairly noisy, 7 . very noisy) for noise annoyance be $y=(1,2$, $\ldots, 7)$. In the research on psychological evaluation for noise annoyance, it has been reported that human can distinguish up to 7 categories when they hear the fluctuating sound (Namba et al., 1978). Each judgement score is affected by the human subjectivity and the borders between two neighboring categories (e.g., noisy and fairly noisy) are vague. Therefore, by regarding the scores as fuzzy observation data and applying fuzzy probability (Zadeh, 1968) to Eq.(1), the probability $\operatorname{Pr}(y=i)$ on the psychological evaluation value $y=i \quad(i=1,2, \ldots, 7)$ can be expressed as:

$$
\operatorname{Pr}(y=i)=\int_{-\infty}^{\infty} m_{i}(x) P(x) d x
$$

where $m_{1}(x)$ denotes the membership function on a typical sound level $x_{1}$ corresponding to the psychological evaluation value $y=i$, and is expressed as follows: 


$$
\begin{aligned}
& m_{1}(x)=\left\{\begin{array}{cc}
1, \quad\left(x<x_{1}\right), \\
e^{-\alpha\left(x-x_{1}\right)^{2}}, & \left(x_{1}<x\right),
\end{array}\right. \\
& m_{i}(x)=e^{-\alpha\left(x-x_{1}\right)^{2}}, \quad(i=2,3, \ldots, 6), \\
& m_{7}(x)=\left\{\begin{array}{cc}
e^{-\alpha\left(x-x_{7}\right)^{2}}, & \left(x<x_{7}\right), \\
1, & \left(x_{7}<x\right),
\end{array}\right.
\end{aligned}
$$

where $\alpha$ is a parameter that determines the shape of the membership function, and can be estimated by use of the observed data. As the membership function $m_{i}(x)$, we have to introduce three kinds of functions as shown in Eq.(6). For two cases when $y=1$ and $y=7$, slightly different functions from the cases when $y=2,3,4,5,6$ have to be considered (cf. Figures 1 and 2).

Substituting for $P(x)$ and $m_{i}(x)$ from Eqs.(1) and (6) into Eq.(5) yields

$$
\begin{aligned}
& \operatorname{Pr}(y=1)=\int_{-\infty}^{x_{1}} P(x) d x+\int_{x_{1}}^{\infty} m_{1}(x) P(x) d x=I_{1}+J\left(x_{1}\right)-K\left(x_{1}\right), \\
& \operatorname{Pr}(y=i)=I_{i},(i=2,3, \ldots, 6), \\
& \operatorname{Pr}(y=7)=\int_{-\infty}^{x_{7}} m_{7}(x) P(x) d x+\int_{x_{7}}^{\infty} P(x) d x=K\left(x_{7}\right)+1-J\left(x_{7}\right)
\end{aligned}
$$

with

$$
\begin{aligned}
& I_{i}=\int_{-\infty}^{\infty} e^{-\alpha\left(x-x_{i}\right)^{2}} P(x) d x, \quad(i=1,2, \ldots, 6), \\
& J\left(x_{1}\right)=\int_{-\infty}^{x_{i}} P(x) d x, \quad(i=1,7), \\
& K\left(x_{i}\right)=\int_{-\infty}^{\alpha_{i}} e^{-\alpha\left(x-x_{i}\right)^{2}} P(x) d x,(i=1,7),
\end{aligned}
$$

By the use of Eq.(1), three functions $I_{i}, J\left(x_{1}\right)$ and $K\left(x_{1}\right)$ can be expressed in forms reflecting the statistics of sound level $x$ (cf. Appendixes 1 and 2).

Furthermore, by applying a fuzzy theory (Tamaki et al., 1992), the conditional probability distribution of sound level $\boldsymbol{x}$ under the condition of the psychological evaluation value $y=i$ is expressed as: 


$$
P(x \mid y=i)=\frac{m_{i}(x) P(x)}{\operatorname{Pr}(y=i)}
$$

Then, the conditional mean can be obtained as

$$
<x \mid y=i>=\frac{\int_{m}^{\infty} x m_{i}(x) P(x) d x}{\operatorname{Pr}(y=i)}
$$

In the same manner as Eqs.(7)-(10), the numerator of Eq.(12) is expressed as

$$
\int_{-\infty}^{\infty} x m_{:}(x) P(x) d x=\left\{\begin{array}{c}
L_{1}+S\left(x_{1}\right)-T\left(x_{1}\right),(i=1), \\
L_{i},(i=2,3, \ldots, 6) \\
T\left(x_{7}\right)+\mu_{x}-S\left(x_{7}\right),(i=7),
\end{array}\right.
$$

with

$$
\begin{aligned}
& L_{i}=\int_{-\infty}^{\infty} x e^{-\alpha\left(x-x_{i}\right)^{2}} P(x) d x, \quad(i=1,2, \ldots, 6), \\
& S\left(x_{i}\right)=\int_{-\infty}^{x_{i}} x P(x) d x,(i=1,7), \\
& T\left(x_{i}\right)=\int_{i 0}^{x_{i}} x e^{-\alpha\left(x-x_{i}\right)^{2}} P(x) d x,(i=1,7),
\end{aligned}
$$

After expanding $x$ in an orthogonal series form:

$$
x=\sum_{j=0}^{1} e_{1 j} H_{j}\left(\frac{x-C_{3}}{\sqrt{1 / 2 C_{1}}}\right),\left(e_{10}=C_{2}, \quad e_{11}=\sqrt{1 / 2 C_{1}}\right),
$$

through the same calculation process as Appendixes 1 and 2, three functions $L_{l}, S\left(x_{t}\right)$ and $T\left(x_{t}\right)$ can be expressed in concrete forms reflecting the statistics of sound level $x$ (cf. Appendix 3).

By putting Eqs.(7), (A5), (A11) and (A12) to the denominator of Eq.(12), and averaging the resultant equation concerning $y$, the mean $\mu_{x}$ of the sound level can be estimated as 


$$
\begin{aligned}
\hat{\mu}_{x} & \equiv \sum_{i=1}^{7}\langle x| y=i>\operatorname{Pr}(y=i)=\langle<x| y=i>_{i} \\
& =<\frac{\int_{-\infty}^{\infty} x m_{i}(x) P(x) d x}{\operatorname{Pr}(y=i)}>_{i} .
\end{aligned}
$$

Similarly, the variance and higher order statistics can be estimated as follows:

$$
\begin{aligned}
& \hat{\sigma}_{x}^{2}=<<\left(x-\mu_{x}\right)^{2} \mid y=i>>, \\
& =<\frac{\int_{-\infty}^{\infty}\left(x-\mu_{x}\right)^{2} m_{i}(x) P(x) d x}{\operatorname{Pr}(y=i)}>_{1}, \\
& \hat{A}_{3} \equiv \frac{1}{\sqrt{3 !}} \ll<H_{2}\left(\frac{x-\mu_{x}}{\sigma_{x}}\right) \mid y=i>_{i} \\
& -\frac{1}{\sqrt{3} !}<\frac{\rho_{+\infty}^{\infty} H_{3}\left(\frac{x-\mu_{x}}{\sigma_{x}}\right) m_{i}(x) P(x) d x}{\operatorname{Pr}(y=i)}>_{i}, \\
& \hat{A}_{4} \equiv \frac{1}{\sqrt{4 !}} \ll<H_{4}\left(\frac{x-\mu_{x}}{\sigma_{x}}\right) \mid y=i>_{i} \\
& =-\frac{1}{\sqrt{4 !}-i}<\frac{\int_{-\infty}^{\infty} H_{i}\left(\frac{x-\mu_{x}}{\sigma_{x}}\right) m_{i}(x) P(x) d x}{\operatorname{Pr}(y=i)}>_{i},
\end{aligned}
$$

where the numerators of Eqs. (19), (20) and (21) can be evaluated through the same calculation process as Eq.(13).

Substituting Eqs.(18)-(21) in Eq.(1), the probability distribution $P(x)$ of the sound level $x$ can be estimated, as :

$$
\left.\dot{P}(x)=\frac{1}{\sqrt{2 \pi \hat{\sigma}_{x}^{2}}} e^{-\frac{\left(x-\hat{i}_{x}\right)^{2}}{2 \hat{\vartheta}_{x}^{2}}}, \mathrm{i}+\sum_{n=1}^{\frac{4}{A_{n}}} \hat{A}_{n} \frac{\frac{1}{\sqrt{n !}}}{\sqrt{n}} H_{n}\left(\frac{\underline{x-\hat{H}_{x}}}{\hat{\sigma}_{x}}\right)\right\},
$$




\section{DYNAMIC STATE ESTIMATION METHOD FOR SOUND ENVIRONMENT SYSTEM BASED ON FUZZY OBSERVATION}

A sound environmental system with power state variables exhibiting a nonGaussian distribution is considered. Let the specific signal power at a discrete time $k$ be $x_{k}$, and expresses the dynamical model for the specific signal as:

$$
x_{k+1}=F x_{k}+v u_{k},
$$

where $u_{k}$ denotes the random input power with known statistics, and $x_{k}$ and $u_{k}$ are uncorrelated to each other. Furthermore, $F$. and $G$ are unknown system parameters and can be estimated by use of the system identification method (Eyhhoff, 1974) when these parameters cannot be determined on the basis of the physical mechanism of system.

The observed data in the actual sound environment often contain the fuzziness due to several causes, for example, the permissible error of the accuracy in measurements, the quantized error in the digitization of observation data, and the variety of human response to the physical stimulus. Therefore in addition to the inevitable external noise, the effects of the fuzziness contained in the observed data have to be first considered in order to derive a state estimation method for the specific signal. The observation equation can be formulated by dividing it into two types of operation from functional viewpoint:

i. The additive property of power state variable, under the existence of external noise:

$$
y_{k}=x_{k}+v_{k},
$$

We assume that the statistics of the external noise power $v_{k}$ are known in advance.

ii. The fuzzy observation $z_{k}$ obtained from $y_{k}$ : The fuzziness of $z_{k}$ is characterized by the membership function $\mu_{z_{k}}\left(y_{k}\right)$.

To derive an estimation algorithm for a specific signal power $x_{k}$, based on the successive observations of fuzzy data $z_{k}$, we focus our attention on Bayes' theorem (Ohta, et al. 1976; Ohta \& Yamada, 1984): 


$$
P\left(x_{k} \mid Z_{k}\right)=\frac{P\left(x_{k}, z_{k} \mid Z_{k-1}\right)}{P\left(z_{k} \mid \bar{z}_{k-1}\right)}
$$

where $Z_{k}\left(=\left(z_{1}, z_{2}, \ldots, z_{k}\right)\right)$ is a set of observation data up to a time $k$. After applying fuzzy probability (Zadeh, 1968) to the right side of Eq.(25), expanding it in a general form of the statistical orthogonal expansion series (Ohta and Nakagami, 1957; Ohta and Koizumi, 1968), the conditional probability density function $P\left(x_{k} \mid Z_{k}\right)$ can be expressed as:

$$
\begin{array}{r}
P\left(x_{k} \mid Z_{k}\right)=\frac{\int_{0}^{\infty} \mu_{z_{k}}\left(y_{k}\right) P\left(x_{k}, y_{k} \mid Z_{k-1}\right) d y_{k}}{\oint_{0}^{\infty} \mu_{z_{k}}\left(y_{k}\right) P\left(y_{k} \mid Z_{k-1}\right) d y_{k}} \\
=\frac{\sum_{m=0}^{\infty} \sum_{n=0}^{\infty} A_{m n} P_{0}\left(x_{k} \mid Z_{k-1}\right) \varphi_{m}^{(1)}\left(x_{k}\right) I_{n}\left(z_{k}\right)}{\sum_{n=0}^{\infty} A_{0 n} I_{n}\left(z_{k}\right)}
\end{array}
$$

with

$$
\begin{aligned}
& I_{n}\left(z_{k}\right)=\oint_{b}^{\infty} \mu_{z_{k}}\left(y_{k}\right) P_{0}\left(y_{k} \mid Z_{k-1}\right) \varphi_{n}^{(2)}\left(y_{k}\right) d y_{k}, \\
& A_{m n}=<\varphi_{m}^{(1)}\left(x_{k}\right) \varphi_{n}^{(2)}\left(y_{k}\right) \mid Z_{k-1}>,
\end{aligned}
$$

The functions $\varphi_{m}^{(1)}\left(x_{k}\right)$ and $\varphi_{i=}^{(2)}\left(y_{k}\right)$ are the orthogonal polynomials of degrees $m$ and $n$ with weighting functions $P_{0}\left(x_{k} \mid Z_{k-1}\right)$ and $P_{0}\left(y_{k} \mid Z_{k-1}\right)$, which can be artificially chosen as the probability density functions describing the dominant parts of $P\left(x_{k} \mid Z_{k-1}\right)$ and $P\left(y_{k} \mid Z_{k-1}\right)$. These two functions must satisfy the following orthonormal relationships:

$$
\begin{aligned}
& \zeta_{0}^{\infty} \varphi_{m}^{(1)}\left(x_{k}\right) \varphi_{m^{\prime}}^{(1)}\left(x_{k}\right) P_{0}\left(x_{k} \mid Z_{k-1}\right) d x_{k}=\delta_{m m^{\prime}}, \\
& \zeta^{\infty} \varphi_{n}^{(2)}\left(y_{k}\right) \varphi_{n^{\prime}}^{(2)}\left(y_{k}\right) P_{0}\left(y_{k} \mid Z_{k-1}\right) d y_{k}=\delta_{n n^{\prime}} .
\end{aligned}
$$

Based on Eq.(26), and using the orthonormal relationship of Eq.(29), the recurrence algorithm for estimating an arbitrary $N$ th order polynomial type function $f_{N}\left(x_{k}\right)$ of the specific signal can be derived as follows: 


$$
\begin{aligned}
\hat{f}_{N}\left(x_{k}\right) & =<f_{N}\left(x_{k}\right) \mid Z_{k}> \\
& =\frac{\sum_{-=n}^{N} \sum_{n=n}^{\infty} A_{m n} C_{N m} I_{n}\left(z_{k}\right)}{\sum_{n=0}^{\infty} A_{0 n} I_{n}\left(z_{k}\right)},
\end{aligned}
$$

where $C_{N m}$ is the expansion coefficient determined by the equality:

$$
f_{N}\left(x_{k}\right)=\sum_{m=0}^{N} C_{N m} \varphi_{m}^{(1)}\left(x_{k}\right)
$$

To make the general theory for estimation algorithm more concrete, the wellknown Gamma distribution is adopted as $P_{0}\left(x_{k} \mid Z_{k-1}\right)$ and $P_{0}\left(y_{k} \mid Z_{k-1}\right)$, because this probability density function is defined within positive region and is suitable to the power state variables.

$$
\begin{aligned}
& F_{0}\left(x_{k} \mid Z_{k-1}\right)=P_{\Gamma}\left(x_{k} ; m_{x_{k}}^{*}, s_{x_{k}}^{\prime}\right) \\
& P_{0}\left(y_{k} \mid Z_{k-1}\right)=P_{\Gamma}\left(y_{k} ; m_{y_{k}}^{*}, s_{y_{k}}^{*}\right)
\end{aligned}
$$

with

$$
\begin{aligned}
& P_{\Gamma}(x ; m, s)=\frac{x^{m-1}}{\Gamma(m) s^{m}} e^{-\frac{x}{s}}, \\
& m_{x_{k}}^{*}=\left(x_{k}^{*}\right)^{2} / \Gamma_{k}, \dot{s}_{x_{k}}^{*}=x_{k}^{*} / m_{x_{k}}^{*}, \\
& \left.x_{k}^{*}=<x_{k}\left|Z_{k-1}>, \Gamma_{k}=<\left(x_{k}-x_{k}^{*}\right)^{2}\right| Z_{k-1}\right\rangle, \\
& m_{y_{k}}^{*}=\left(y_{k}^{*}\right)^{2} / \Omega_{k}, \quad s_{y_{k}}^{*}=y_{k}^{*} / m_{y_{k}}^{*}, \\
& y_{k}^{*}=<y_{k}\left|Z_{k-1}>=x_{k}^{*}+<v_{k}\right\rangle, \\
& \Omega_{k}=<\left(y_{k}-y_{k}^{*}\right)^{2} \mid Z_{k-1}>=\Gamma_{k}+\left\langle\left(v_{k}-\left\langle v_{k}\right\rangle\right)^{2}\right\rangle
\end{aligned}
$$


Then, the orthonormal functions with two weighting probability density functions in Eq.(33) can be given in Lagguer polynomial (Ohta \& Nakagami, 1957; Ohta \& Koizumi, 1968):

$$
\begin{aligned}
& \varphi_{m}^{(1)}\left(x_{k}\right)=\sqrt{\frac{\Gamma\left(m_{x_{k}}^{*}\right) m !}{\Gamma\left(m_{x_{k}}^{*}+m\right)} L_{m}^{\left(m_{x_{k}}^{*}-1\right)}\left(\frac{x_{k}}{s_{x_{k}}^{*}}\right),} \\
& \varphi_{\eta}^{(2)}\left(y_{k}\right)=\sqrt{\frac{\Gamma\left(m_{y_{k}}^{*}\right) n !}{\Gamma\left(m_{y_{k}}^{*}+n\right)} L_{:}^{\left(m_{y_{k}}^{*}-1\right)}\left(\frac{y_{k}}{s_{y_{k}}^{*}}\right),}
\end{aligned}
$$

where the Lagguer polynomial $L_{n}^{(m)}(x)$ is defined by

$$
L_{: a}^{(m)}(x)=\frac{e^{x} x^{-m}}{n !} \frac{d^{n}}{d x^{n}}\left(e^{-x} x^{n+m}\right)
$$

As the membership function $\mu_{z_{k}}\left(y_{k}\right)$, the following function suitable for the Gamma distribution is adopted.

$$
\mu_{z_{k}}\left(y_{k}\right)=\left(z_{k}^{-\beta} e^{\beta}\right) y_{k}^{\beta} \exp \left\{-\frac{\beta}{z_{k}} y_{k}\right\},
$$

where $\beta(>0)$ is a parameter. Accordingly, Eq.(27) can be given by (cf. Appendix 4)

$$
I_{n}\left(z_{k}\right)=\frac{z_{k}^{-\beta} e^{\beta}}{\Gamma\left(m_{y_{k}}^{*}\right)\left(s_{y_{k}}^{*}\right)^{m_{y_{k}}}} \Gamma\left(M_{k}\right) D_{-}^{M_{k}} \sqrt{\frac{\Gamma\left(m_{y_{k}}^{*}\right) n !}{\Gamma\left(m_{y_{k}}^{*}+n\right)}} g_{n 0}
$$

with

$$
M_{k}=m_{y_{k}}^{*}+\beta, D_{k}=\frac{s_{y_{k}}^{*} z_{k}}{\beta s_{y_{k}}^{*}+z_{k}},
$$

where a few concrete expressions of $g_{n 0}$ in Eq.(38) can be expressed as follows:

$$
\begin{aligned}
& g_{00}=1, \\
& g_{10}=\dot{m_{y_{k}}}-M_{k} \frac{D_{k}}{s_{y_{k}}^{*}}, \\
& g_{20}=\frac{1}{2}\left(m_{y_{k}}^{*}+1\right) m_{y_{k}}^{*}-\left(m_{y_{k}}^{*}+1\right) M_{k} \frac{I_{k}}{\dot{s_{y_{k}}}}+\frac{1}{2}\left(1+M_{k}\right) M_{k}\left(\frac{D_{k}}{s_{y_{k}}^{*}}\right)^{2}, \\
& g_{30}=\frac{1}{6}\left(m_{y_{k}}^{*}+2\right)\left(m_{y_{k}}^{*}+1\right) m_{y_{k}}^{*}-\frac{1}{2}\left(m_{y_{k}}^{\dot{*}}+2\right)\left(\dot{m_{y_{k}}}+1\right) M_{k} \frac{D_{k}}{\dot{s_{y_{k}}}}
\end{aligned}
$$




$$
+\frac{1}{2}\left(m_{y_{k}}^{*}+2\right)\left(1+M_{k}\right) M_{k}\left(\frac{D_{k}}{s_{y_{k}}^{*}}\right)^{2}-\frac{1}{6}\left(2+M_{k}\right)\left(1+M_{k}\right) M_{k}\left(\frac{D_{k}}{s_{y_{k}}^{*}}\right)^{3} .
$$

Especially, by considering two special cases with

$$
f_{1}\left(x_{k}\right)=x_{k}, f_{2}\left(x_{k}\right)=\left(x_{k}-\hat{x}_{k}\right)^{2}
$$

in Eq.(31), the estimates for mean and variance can be obtained as follows:

$$
\begin{aligned}
& \hat{x}_{k}=<x_{k} \mid Z_{k}> \\
& =\frac{\sum_{n=0}^{\infty}\left\{A_{0 n} C_{10}+A_{1 n} C_{11}\right\} I_{n}\left(z_{k}\right)}{\sum_{n=0}^{\infty} A_{0 n} I_{n}\left(z_{k}\right)}, \\
& P_{k}=<\left(x_{k}-\hat{x}_{k}\right)^{2}\left|Z_{k}\right\rangle \\
& =\frac{\sum_{n=0}^{\infty}\left\{A_{o n} C_{20}+A_{1 n} C_{21}+A_{2 n} C_{22}\right\} I_{n}\left(z_{k}\right)}{\sum_{n=0}^{\infty} A_{0 n} I_{n}\left(z_{k}\right)}
\end{aligned}
$$

with

$$
\begin{aligned}
& C_{10}=m_{x_{k}}^{*} s_{x_{k}}^{*}, \\
& C_{11}=-\sqrt{m_{x_{k}}^{*}} s_{x_{k}}^{*}, \\
& C_{20}=\hat{x}_{k}^{2}-2 m_{x_{k}}^{*} s_{x_{k}}^{*}\left\{\hat{x}_{k}-\left(m_{x_{k}}^{*}+1\right) s_{x_{2}}^{*}\right\}-m_{x_{k}}^{*}\left(m_{x_{k}}^{*}+i\right) s_{x_{k}}^{*}, \\
& C_{21}=2 \sqrt{m_{x_{2}}^{*}} s_{x_{2}}^{*}\left\{\dot{x}_{k}-\left(m_{x_{k}}^{*}+1\right) s_{x_{k}}^{*}\right\}, \\
& C_{22}=\sqrt{\left.2 m_{\dot{x}_{k}}^{*} ! m_{\tilde{n}_{k}}^{*}+1\right)} s_{x_{k}}^{*},
\end{aligned}
$$

where, by considering Eq.(24), some of the expansion coefficients defined by Eq.(28) can be expressed in a form reflecting the statistics of the external noise power, as follows: 


$$
\begin{aligned}
& A_{00}=1, \quad A_{10}=A_{01}=A_{20}=A_{02}=A_{21}=0, \\
& A_{11}=\sqrt{\frac{m_{x_{k}}^{*}}{m_{y_{k}}^{*}} \frac{s_{x_{k}}^{*}}{s_{y_{k}}^{*}},} \\
& A_{12}=\frac{s_{x_{k}}^{*}}{s_{y_{k}}^{*}} \sqrt{\frac{2 m_{x_{k}}^{*}}{m_{y_{k}}^{*}\left(m_{y_{k}}^{*}+1\right)}}\left\{\left(m_{y_{k}}^{*}+1\right) m_{y_{k}}^{*}-\frac{\left\langle v_{k}\right\rangle}{s_{y_{k}}^{*}}-\left(m_{x_{k}}^{*}+1\right) \frac{s_{x_{k}}^{*}}{s_{y_{k}}^{*}}\right\}, \\
& A_{22}=\sqrt{\frac{\left(m_{x_{k}}^{*}+1\right) m_{x_{k}}^{*}}{\left(m_{y_{k}}^{*}+1\right) m_{y_{k}}^{*}}\left(\frac{s_{x_{k}}^{*}}{s_{y_{k}}^{*}}\right)^{2} .}
\end{aligned}
$$

Finally, by considering Eq.(23), the prediction step which is essential to perform the recurrence estimation can be given by

$$
\begin{aligned}
& \left.x_{k+1}^{*}=F \hat{x}_{k}+G<u_{k}\right\rangle, \\
& \Gamma_{k+1}=F^{2} P_{k}+G^{2}<\left(u_{k}-\left\langle u_{k}>\right)^{2}\right\rangle
\end{aligned}
$$

By replacing $k$ with $k+1$, the recurrence estimation can be achieved.

\section{APPLICATION TO SOUND ENVIRONMENT}

To examine the practical usefulness of the proposed static and dynamic state estimation methods based on fuzzy observation, the proposed methods are applied to the actual sound environment. The road traffic noise is adopted as an example of a specific signal with a complex fluctuation form.

We measured the road traffic noise data in two typical situations of heavy traffic flow and light traffic flow, and the sound level data with mean values 80.2 [dB] and $71.4[\mathrm{~dB}]$ were used in the experiment. We call these data as data 1 and data 2 respectively. After recording a road traffic noise into a data recorder, replaying it in a laboratory room, 6 subjects with normal hearing ability evaluated with 7 noise annoyance scores from (1) very calm to (7) very noisy at every 5 seconds. To apply the proposed static estimation method in Section 2 to the actual data, it is first necessary to decide the parameter $\alpha$ in the membership function of Eq.(6). The data 1 composed of a pair of data on the sound level and the corresponding noise 
annoyance, was utilized to estimate the parameter as the training data. By use of the training data, the parameter was decided so as to satisfy a pair of data as accurately as possible. The membership functions obtained from the experimental data are shown in Figure 1 (for data 1) and Figure 2 (for data 2) respectively. Next, by use of

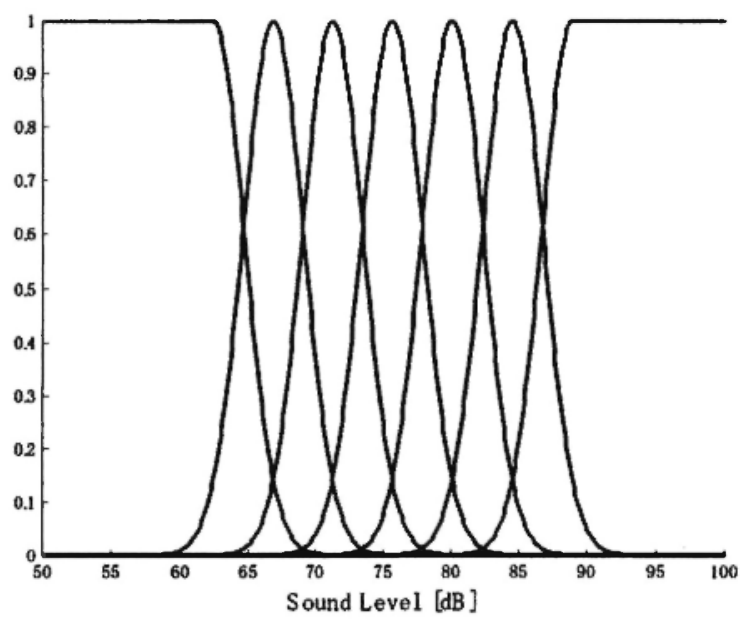

Fig. 1: Membership function for data 1

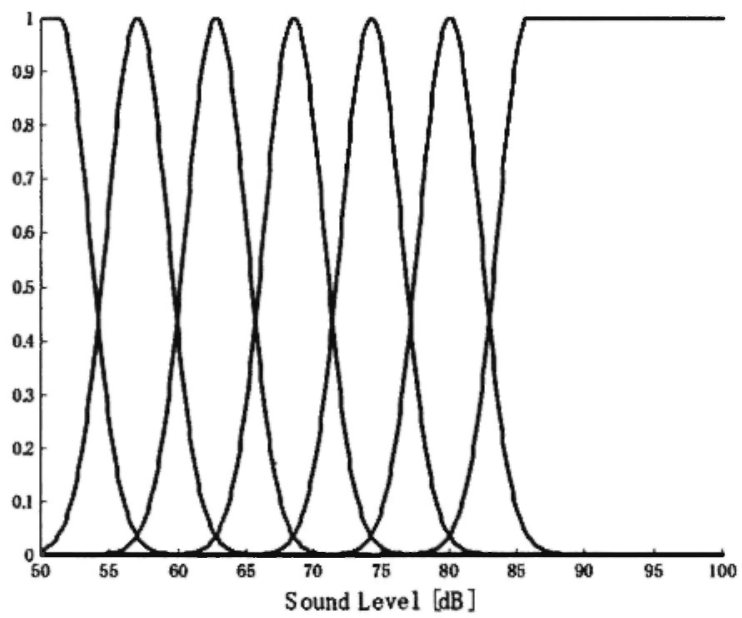

Fig. 2: Membership function for data 2 
membership function with the estimated parameter, the probability distribution of the sound level for the data 2 was estimated on the basis of the noise annoyance data from Eqs.(18) to (22).

A theoretically estimated curve for the cumulative distribution of sound level is shown in figure 3 which is estimated by considering fuzzy theory based on the training data for a subject. In this figure, the theoretical curve with the $1^{\text {st }}$ approximation was considered the only first term in Eq.(22) (i.e., Gaussian distribution). The $2^{\text {nd }}$ and $3^{\text {rd }}$ approximations in these figures were considered the expansion terms up to $\hat{A}_{3}, \hat{A}_{4}$ respectively. The theoretical curves show good agreements with experimental sampled points by considering the expansion coefficients with several higher orders. By estimating once the parameter of membership function based on arbitrary training data, the probability distribution on the sound level with quite different statistics from the training data can be estimated by use of the proposed method.

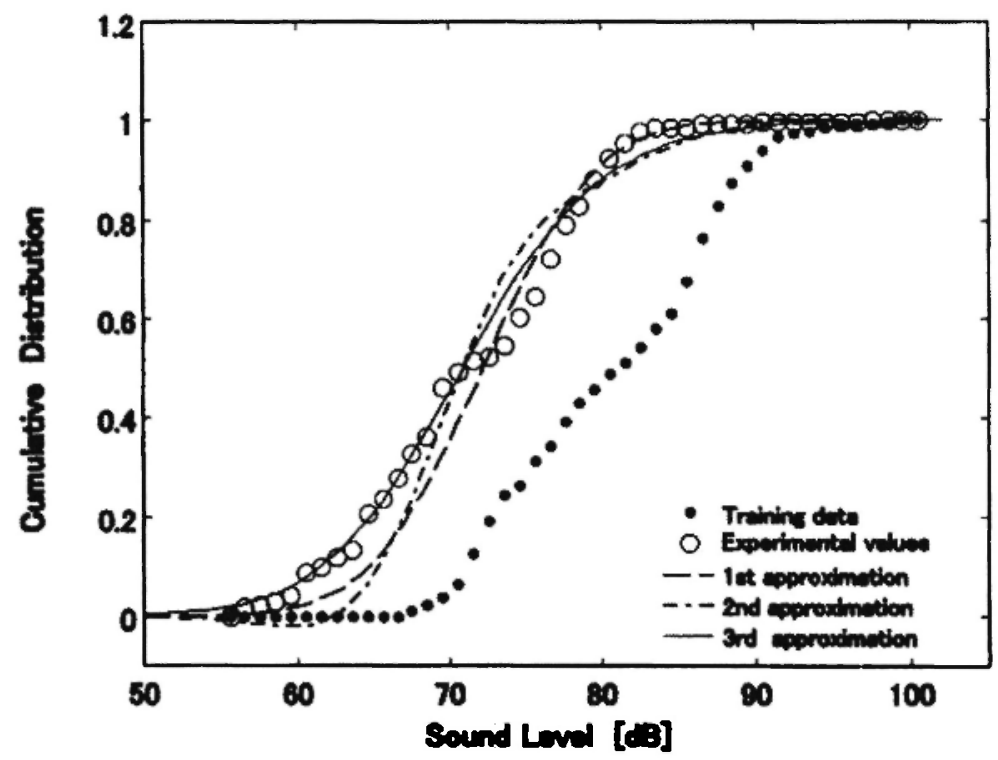

Fig. 3: An estimation result for the cumulative distribution of sound level by considering fuzzy theory based on the training data for a subject 


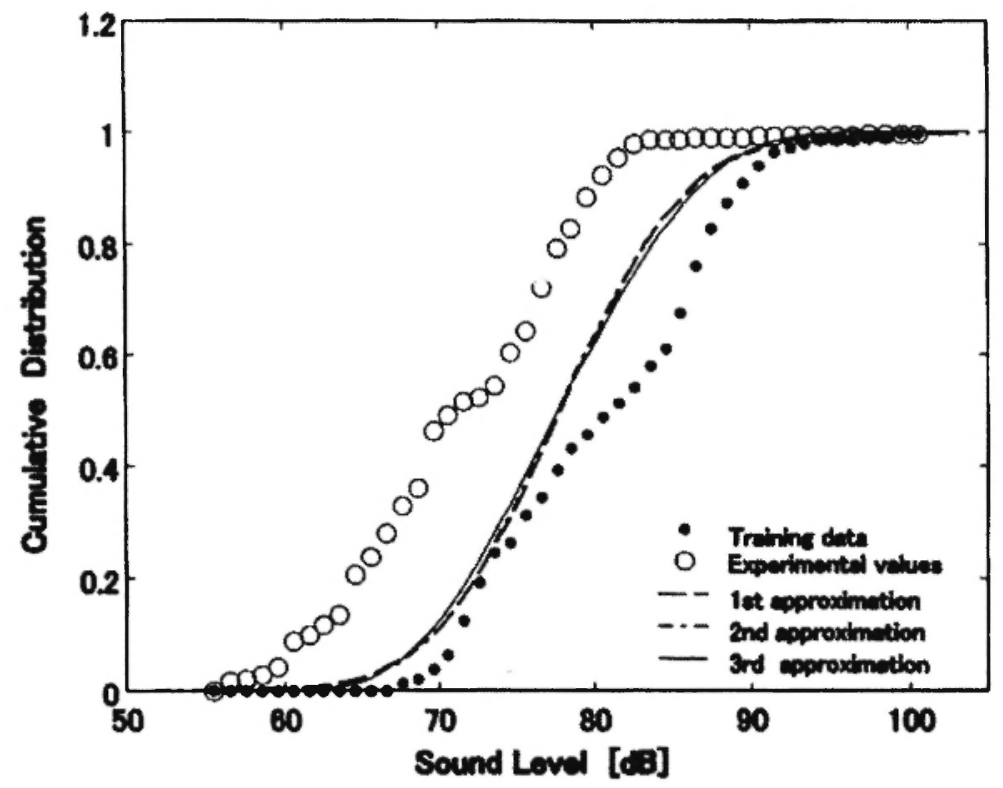

Fig. 4: An estimation result for the cumulative distribution of sound level without considering fuzzy theory based on the training data for a subject

For comparison, the estimated result based on the extended regression analysis method reported in our previous paper (Ohta and Ikuta, 1988; Ohta and Ikuta, 1995), without considering fuzzy theory, is shown in figure 4. There are great discrepancies between the theoretical and experimental values in this figure. These results clearly show the effectiveness of the proposed method by introducing the fuzzy theory.

The proposed dynamic estimation method described in Section 3 was next applied to the actual observed data, which were contaminated by background noise and quantized with $1 \mathrm{~dB}$ width and roughly with $2 \mathrm{~dB}$ width. Then the fluctuation wave form of the specific signal (i.e., road traffic noise) was estimated. A white noise was adopted as a background noise. The road traffic noise and white noise were respectively measured by use of a sound level meter and recorded into a data recorder. After replaying the recorded specific signal and the background noise simultaneously in an anechoic room, by quantizing the observation data measured in the anechoic room, fuzzy observation data were obtained. The quantized observation data were considered after contamination by the background noise as observation of fuzzy data, and the proposed dynamic estimation method was applied. 
Figures 5 and 6 show the estimation results of the fluctuation wave form of the specific signal. In this estimation, the finite number of expansion coefficients $A_{m n}(m, n \leq 2)$ was used for the simplification of the estimation algorithm in Eqs. (41) and (42). In these figures, the horizontal axis shows the discrete time $k$, of the estimation process, and the vertical axis expresses the sound level taking a logarithmic transformation of power-scaled variables, because the actual sound environment usually is evaluated on $\mathrm{dB}$ scale connected with human effects. For comparison, the estimation results calculated using the usual method without considering any membership function are also shown in these figures. Since Kalman's filtering theory is widely used in the field of stochastic system (Kalman, 1960; Kalman \& Bucy, 1961), this method was also applied to the fuzzy observation data as a trail. The results estimated by the proposed method considering the membership function show good agreement with the true values. On the other hand, there are great discrepancies between the estimates based on the standard type dynamical estimation method (i.e., Kalman filter) without consideration of the membership function and the true values, particularly in the estimation of the lower level values of the fluctuation.

\section{CONCLUSION}

In this study, based on the observed data containing fuzziness, two types of state estimation methods of a specific signal have been proposed from static and dynamic viewpoints. More specifically, by introducing fuzzy probability, a static estimation method for the probability density function of the sound level has been derived on the basis of the evaluation of noise annoyance. Next, a dynamical method for estimating the fluctuation wave form of a specific signal has been proposed by paying our attention to the power state variable satisfying the additive property of the specific signal and the external noise. The proposed dynamical estimation method has been realized by introducing a new type of membership function suitable for the power state variable and applying the fuzzy probability. The proposed methods have been applied to the actual estimation problems in a sound environment system, and it has been experimentally verified that better results have certainly been obtained than the results employing usual methods without considering any membership function. 


\section{N. Hayakawa}

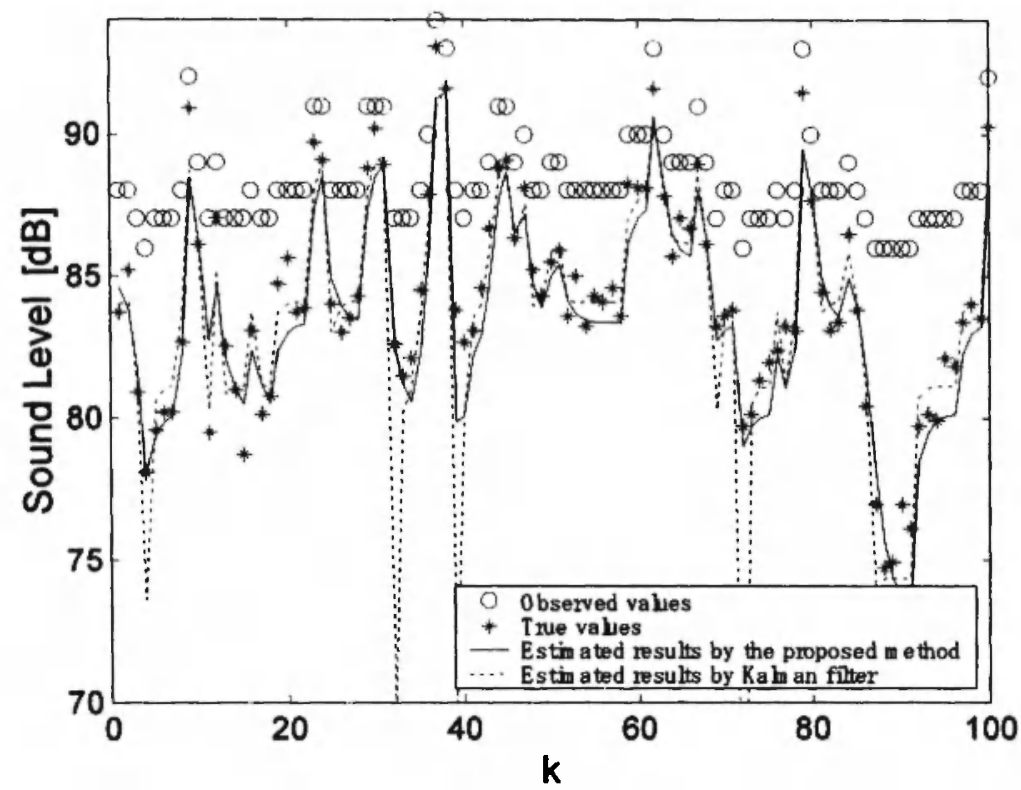

Fig. 5: State estimation results for the road traffic noise based on the observed data contaminated by background noise and quantized with $1 \mathrm{~dB}$ width.

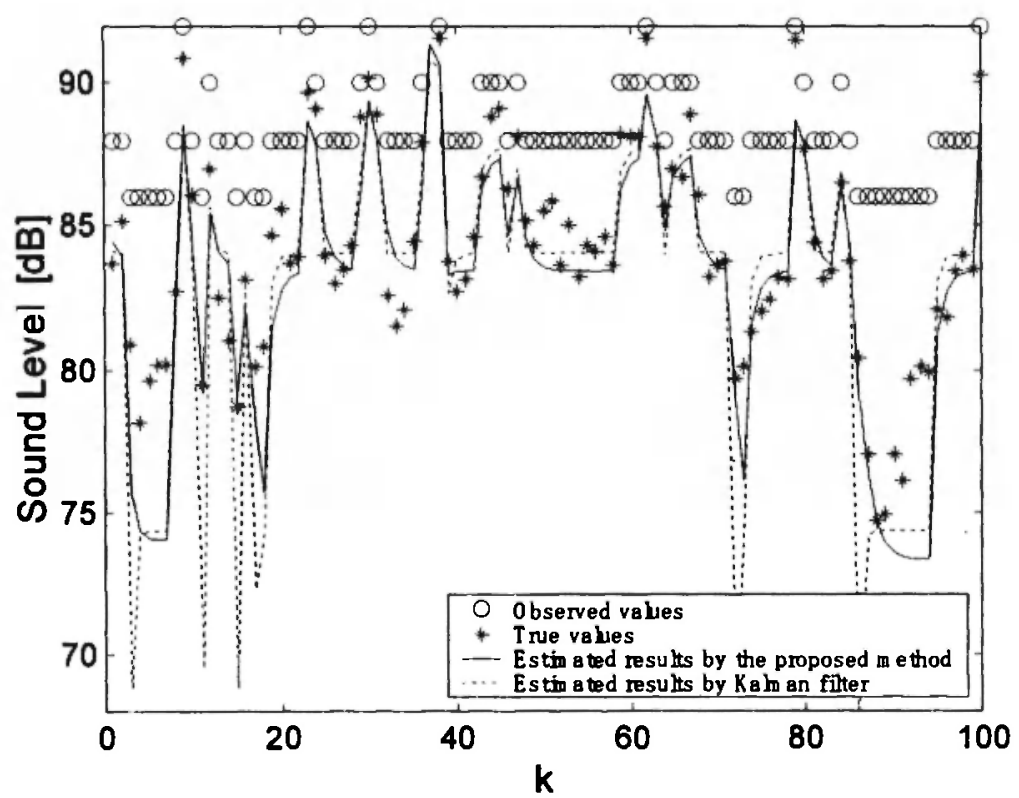

Fig. 6: State estimation results for the road traffic noise based on the observed data contaminated by background noise and quantized with $2 \mathrm{~dB}$ width. 
The proposed method is quite different from the traditional standard approach. However, it is still at its early stage of study, and there are a number of practical problems to be explored in the future, starting from the results of the basic study in this paper. Some of the problems are the following.

i. The proposed static and dynamic methods should be applied to the other actual data of sound environment systems, and the practical usefulness should be verified in each actual situation.

ii. The proposed static theory should be further extended into more realistic cases in the presence of background noise.

iii. The proposed dynamical method should be extended to cases with multidimensional state variable and multi-source configurations.

\section{REFERENCES}

Cramer, H. 1951. Mathematical Methods of Statistics, Princeton, Princeton University Press, 133, 221-7.

Eyhhoff, P. 1974. System Identification: parameter and state estimation, New York, John Wiley \& Sons.

Kalman, R.E. 1960. A new approach to linear filtering and prediction problems, Transactions on ASME, Series D, Journal of Basic Engineering, 82, 35-45.

Kalman, R.E., and Bucy, R. 1961. New results in linear filtering and prediction theory, Transactions on ASME, Series D, Journal of Basic Engineering, 83, 95-108.

Kushner, H.J. 1967. Approximations to optimal nonlinear filters, IEEE Transactions on Automatic Control, AC-12, 546-556.

Namba, S., Kuwano, S., and Nakamura, T. 1978. Rating of road traffic noise using the method of continuous judgment by category, The Journal of The Acoustical Society of Japan, 34, 29-34. [in Japanese]

Ohta, M. and Nakagami, M. 1957. On the general energy distribution of the brownian motion, Proceedings of the $7^{\text {th }}$ Japan National Congress for Applied Mechanics, 317-22.

Ohta, M. and Koizumi, T. 1968. General treatment of the response of a nonlinear rectifying device to a stationary random input, IEEE Transactions on Information Theory, IT-14, 595-8.

Ohta, M., Hatakeyama, K., and Nishimura, M. 1976. Some unified methods in multivariate linear filtering and prediction problems by use of the expansion form of Bayes' theorem, Proceedings of the $26^{\text {th }}$ Japan National Congress for Applied Mechanics, 383-98. 
Ohta, M. and Yamada, H. 1984. New methodological trials of dynamical state estimation for the noise and vibration environmental system: establishment of general theory and its application to urban noise problems, Acustica, 55, 199-212.

Ohta, M. and Ikuta, A. 1988. A New trial of regression analysis with less information loss and its application to sound insulation walls: matching to the prediction of response probability distribution, The Journal of The Acoustical Society of Japan, 44, 848-56. [in Japanese]

Ohta, M. and Ikuta, A. 1995. An acoustic signal processing for generalized regression analysis with reduced information loss based on data observed with amplitude limitation, Acustica, 81, 129-35.

Tamaki, F., Kanagawa, A. and Ohta, H. 1992. Estimation of population distribution based on fuzzy observation data, Journal of Japan Society on Fuzzy Theory and Systems, 4, 114-19. [in Japanese]

Zadeh, L.A. 1968. Probability measure of fuzzy events, Journal of Mathematical Anatysis and Applications, 23, 421-7.

\section{APPENDIX 1: EXPRESSION OF EQ. (8)}

Substituting Eq. (1) into Eq. (8) and considering the relations: $H_{0}(x)=1$ and $A_{0}=1$, the following expression is obtained.

$$
\begin{gathered}
I_{i}=\int_{-\infty}^{\infty} \frac{1}{\sqrt{2 \pi \sigma_{x}^{2}}} e^{-\left\{\alpha\left(x-x_{i}\right)^{2}+\left(x-\mu_{x}\right)^{2} \cdot 2 \sigma_{x}^{2}\right\}} \sum_{n=0}^{\infty} A_{n} \frac{1}{\sqrt{n !}} H_{n}\left(\frac{x-\mu_{x}}{\sigma_{x}}\right) d x \\
=\frac{e^{C_{3}}}{\sqrt{2 C_{1} \sigma_{x}^{2}}} \int \frac{1}{\sqrt{\pi / C_{1}}} e^{-\frac{\left(x-C_{2}\right)^{2}}{1 / C_{1}}} \sum_{n=0}^{\infty} \sum_{r=0}^{n} A_{n} \frac{1}{\sqrt{n !}} d_{n r} H_{r}\left(\frac{x-C_{2}}{\sqrt{1 / 2 C_{1}}}\right) d x
\end{gathered}
$$

with

$$
C_{1}=\frac{2 \alpha \sigma_{x}^{2}+1}{2 \sigma_{x}^{2}}, C_{2}=\frac{2 \alpha \sigma_{x}^{2} x_{i}+\mu_{x}}{2 \alpha \sigma_{x}^{2}+1}, C_{3}=C_{1}\left(C_{2}{ }^{2}-\frac{2 \alpha \sigma_{x}^{2} x_{i}^{2}+\mu_{x}^{2}}{2 \alpha \sigma_{x}^{2}+1}\right),
$$

where $d_{n r} r=0,1,2, \ldots, n$ represent the expansion coefficients in the equality.

$$
H_{n}\left(\frac{x-\mu_{x}}{\sigma_{x}}\right)=\sum_{r=0}^{n} d_{n r} H_{r}\left(\frac{x-C_{2}}{\sqrt{1 / 2 C_{1}}}\right) \text {. }
$$


Considering a relationship:

$$
\left.\int_{-\infty}^{\infty} \frac{1}{\sqrt{\pi / C_{1}}} \div \frac{\left(x-C_{2}\right)^{2}}{1 / C_{1}}, \frac{x-C_{2}}{\sqrt{1 / 2 C_{1}}} ; \frac{1}{\sqrt{r !}} ; \frac{x-C_{2}}{\sqrt{1 / 2 C_{1}}}\right) d x=\delta_{0 r},
$$

which is a special case of Eq. (3), Eq. (Al) can be expressed as:

$$
I_{l}=\frac{e^{C_{3}}}{\sqrt{2 C_{1} \sigma_{x}^{2}}} \sum_{n=0}^{\infty} A_{n} \frac{1}{\sqrt{n !}} d_{n 0}
$$

\section{APPENDIX 2: EXPRESSIONS OF EQS. (9) AND (10)}

After substituting Eq. (1) into Eq. (9), transforming the variable: $z=\left(x-\mu_{x}\right) / \sigma_{x}$, the following relationship is obtained.

$$
J\left(x_{i}\right)=\int_{-\infty}^{\left.x_{i}-\mu_{x}\right) / \sigma_{x}} \frac{1}{\sqrt{2 \pi}} e^{-\frac{z^{2}}{2}}\left\{1+\sum_{n=1}^{\infty} A_{n} \frac{1}{\sqrt{n !}} H_{n}(z)\right\} d z
$$

By using the definition of Hermite polynomial of Eq. (2), Eq. (A6) is expressed as follows:

$$
\begin{aligned}
& J\left(x_{i}\right)=\Phi\left(\frac{x-11}{\sigma_{x}}\right)+\sum_{n=1}^{\infty} A_{n} \frac{-1}{\sqrt{2 \pi n !}}(-1): l \frac{d^{n-1}}{d z^{n} !} e^{-\frac{z^{2}}{2}} J_{-\infty}^{\prime} \ddot{n}_{x}, z_{x}, \\
& \Phi(X)=\int_{-\infty}^{X} N(x) d x, \quad N(x)=\frac{1}{\sqrt{2 \pi}} e^{-\frac{x^{2}}{2}}
\end{aligned}
$$

By considering the condition:

$$
\lim _{z \rightarrow \pm \infty} \frac{u^{n}}{d z^{n}} e^{-\frac{z^{2}}{2}}=0
$$

and using again the definition of Hermite polynomial, the following relationship is derived: 


$$
J\left(x_{i}\right)=\underline{\underline{u}}, \frac{x_{i}-\mu_{x}}{\sigma_{x}} ;-\sum_{n=1}^{\infty} A_{n}-\frac{1}{\sqrt{n !}} N\left(\frac{x_{i}-\mu_{x}}{\sigma_{x}}\right) H_{: . .} !\left(\frac{x_{i}-\mu_{x}}{\sigma_{x}}\right) .
$$

Furthermore, based on Eq. 1, through the same caluculation process as the derivation of Eq. (A10), the function $K\left(x_{i}\right)$ in $\mathrm{Eq} .(10)$ is given by

$$
\left.K\left(x_{i}\right)=\frac{e^{C_{3}}}{\sqrt{2 C_{1} \sigma_{x}^{2}}} ; \frac{x_{i}-C_{2}}{\sqrt{1 / 2 C_{1}}} ;-\sum_{n=1}^{\infty} \sum_{r=1}^{n} A_{n} \frac{1}{\sqrt{n !}}-N\left(\frac{x_{i}-C_{2}}{\sqrt{1 / 2 C_{1}}}\right) H_{r}-i\left(\frac{x_{i}-C_{2}}{\sqrt{1 / 2 C_{1}}}\right)\right\} .
$$

APPENDIX 3: EXPRESSIONS OF FUNCTIONS $L_{i}, S\left(x_{i}\right)$ AND $T\left(x_{i}\right)$

$$
\begin{aligned}
& L_{i}=\frac{e^{C_{3}}}{\sqrt{2 C_{1} \sigma_{x}^{2}}} \sum_{r=0}^{1} \sum_{n=r}^{\infty} A_{n} \frac{1}{\sqrt{n !}} e_{1 r} d_{n r}, \\
& S\left(x_{i}\right)=\Phi\left(\frac{x_{i}-\mu_{x}}{\sigma_{x}}\right)-f_{11} N\left(\frac{x_{i}-\mu_{x}}{\sigma_{r}}\right) \\
& -\sum_{n=1}^{\infty} \sum_{j=0}^{n+1} \frac{1}{\sqrt{n !}} A_{n ! n} f_{n: 1, j} N\left(\frac{x_{i}-\mu_{x}}{\sigma_{x}}\right) H_{i=1}\left(\frac{x_{i}-\mu_{x}}{\sigma_{x}}\right), \\
& T\left(x_{1}\right)=\frac{e^{C_{3}}}{\sqrt{2 C_{1} \sigma_{x}^{2}}}\left\{\Phi\left(\frac{x_{i}-C_{2}}{\sqrt{1 / 2 C_{1}}}\right)-\bar{s}_{11} N\left(\frac{x_{i}-C_{2}}{\sqrt{1 / 2 C_{1}}}\right)\right. \\
& \left.-\sum_{n=1}^{\infty} \sum_{j=0}^{n+1} \frac{1}{\sqrt{n !}} A_{n} g_{n+1, j} N\left(\frac{x_{i}-C_{2}}{\sqrt{1 / 2 C_{1}}}\right) H_{j-1}\left(\frac{x_{i}-C_{2}}{\sqrt{1 / 2 C_{1}}}\right)\right\},
\end{aligned}
$$

where $f_{n+1}$, and $g_{n+1, j}$ are coefficients satisfying the following equality:

$$
x H_{n}\left(\frac{x-\mu_{x}}{\sigma_{x}}\right)=\sum_{j=0}^{n+1} f_{n+1, i} H_{i}\left(\frac{x-\mu_{x}}{\sigma_{x}}\right)=\sum_{j=0}^{n+1} g_{n+1, j} H_{j}\left(\frac{x-C_{2}}{\sqrt{1 / 2 C_{1}}}\right) .
$$

